\title{
QUANTITATIVE SPECTROSCOPY OF WOLF-RAYET STARS
}

\author{
W. Schmutz \\ Institut für Theoretische Physik und Sternwarte der Universität Kiel \\ 0lshausenstrasse, D-2300 Kiel 1, Federal Republic of Germany
}

\section{INTRODUCTION}

Advances in theoretical modeling of rapidly expanding atmospheres in the past few years made it possible to determine the stellar parameters of the Wolf-Rayet stars. This progress is mainly due to the improvement of the models with respect to their spatial extension: The new generation of models treat spherically-symmetric expanding atmospheres, i.e. the models are one-dimensional. 0lder models describe the wind by only one representative point. The older models are in fact 'core-halo' approximations. They have been introduced by Castor and van Blerkom (1970), and were extensively employed in the past (cf. e.g. Willis and Wilson, 1978; Smith and Willis, 1982). First results from new one-dimensional model calculations are published by Hillier (1984), Schmutz (1984), Hamann (1985), Hillier (1986), and Schmutz et al. (1987a); more detailed results are presented by Schmutz and Hamann (1986), Hamann and Schmutz (1987), Hillier (1987a,b), Wessolowski et al. (1987), Hillier (1987c) and Hamann et al. (1987). These results demonstrate that the step from zero- to one-dimensional calculations is essential. The important point is that the complicated interrelation between NLTE-level populations and radiation field is treated adequately (Schmutz and Hamann, 1986; Hillier, 1987). For this interrelation it is crucial to model consistently not only the line-formation region, but also the layers where the continuum is emitted. In fact, it is the core-halo approximation that causes the one-point models to fail in certain aspects.

\section{MODEL CALCULATIONS}

Presently, there are two different codes that are able to model adequately wolfRayet atmospheres: the model of the Kiel group and Hillier's model. The two models are completely different in their technical approach how the NLTE-problem is solved (Hamann, 1986; Hillier, 1987a). Nevertheless, both models yield essentially the same results - an encouraging fact. Both models are founded on similar physical assumptions: The velocity law is predefined analytically and the temperature 
stratification is determined assuming radiative equilibrium. Up to now, the kielcalculations are for a pure helium atmosphere, while Hillier (1987c) includes in addition hydrogen as well as nitrogen and carbon in an approximate way.

The model-input parameters are $T_{\star}, R_{\star}$ and $\dot{M}$; the informations provided by the calculations which can be compared with the observations are the emergent line profiles and the continuum fluxes.

\section{EFFECTIVE TEMPERATURE AND $T_{\star}$}

In a spherically-extended stellar atmosphere, the reference radius has to be defined to which the effective temperature is referred. This may be, e.g., $R_{2 / 3}$, the radius where the Rosseland optical depth becomes $2 / 3$. However, in order to compare the temperature resulting from a spectral analysis with the predictions of the stellar evolution calculations, the temperature should be referred to a fictitious "hydrostatic" radius. The model calculations provide the radius at the base of the model-wind, termed "core radius", $R_{\star}$, and the temperature referred to this coreradius, $T_{\star}$. The core-radius, $R_{\star}$, is the best available approximation to the hydrostatic radius. It has to be admitted, however, that if the continuum optical depth is of the order unity at layers where the wind has reached a velocity which is a considerable fraction of its terminal velocity, the core radius results from an inward extrapolation of the velocity field into regions that are not accessible to the observations. Thus, the derived core radius depends on the adopted velocity law. Luckily, it turns out that for most (about 70\%) of the Wolf-Rayet stars the difference between $R_{2 / 3}$ and $R_{\star}$ is small, i.e. the resulting differences between $T_{\text {eff }}\left(R_{2 / 3}\right)$ and $T_{*}$ are comparable to the uncertainties of the derived temperature. Moreover, test calculations for a Wolf-Rayet star with an extended atmosphere (WR136 - Wessolowski et al., in preparation) showed that the resulting stellar parameters $R_{*}$ and $T_{*}$ do not depend dramatically on the adopted velocity law. Hence, the stellar parameters $T_{\star}$ and $R_{\star}$ can be compared with the results of the stellar evolution theory without introducing a large ambiguity.

\section{DIAGNOSTIC OF SPECTRAL LINES}

It can be shown that all models for which the parameters $\dot{M}, R_{\star}$ and $V_{\text {inf }}$ combine to the same value of the parameter $p^{W}$, yield, to a good degree of approximation, the same (helium-) line equivalent widths. The parameter $p^{W}$ is defined by

$$
p^{W}=\frac{\dot{M}}{v_{\text {inf }} R_{\star}^{3 / 2}}
$$


This homologous-atmosphere parameter may be termed "wind density", though its physical dimension is not a density. The wind density and the effective temperature are the fundamental parameters of a Wolf-Rayet spectrum - analog to $T_{\text {eff }}$ and $g$ in stars with static atmospheres. In other words, for Wolf-Rayet atmospheres the wind density plays the role which has the gravity in static atmospheres. In contrast to the gravity in static atmospheres, however, the wind density cannot be linked with other fundamental stellar parameters. The obvious reason is that the mass-loss mechanism acting in Wolf-Rayet stars is not identified. In the cases when radiation pressure is the driving force of the wind, the wind density is linked to the ratio of radiation forces to gravity, and is hence, a function of mass, radius and luminosity. A minor difference to the gravity-analog in static atmospheres is, that the wind density is only an approximate parameter: i.e. models with the same parameter-pair, $T_{*}, p^{W}$, are not exactly homologous. E.g., for the He I equivalent widths, $p^{W}$ depends on a slightly different power of $R_{*}, p^{W}\left(\right.$ He I) $\sim R_{\star}^{-5 / 3}$, than for the He II lines, $p^{W}$ (He II) $\sim R_{\star}^{-3 / 2}$ (cf. Eq. 1).

\section{SPECTRAL CLASSIFICATION}

The Wolf-Rayet classification is a one-dimensional system. However, as outlined in Sect. 4, their spectra depend on two parameters. Therefore, a two-dimensional classification clearly has to be introduced, e.g. as aiready suggested by Hiltner and Schild (1966) and refined by Walborn (1974). For later use in this paper, we divide the Wolf-Rayet stars into four classes:

\section{WNE-A WNE-B WNL WC.}

Tentatively, we employ as criterion to separate "A" from "B" class the strength of the He II line at $5412 \AA$ : if its equivalent width is less than $40 \AA$, the star is assigned to the " $A$ " class, otherwise to the "B" class.

\section{WOLF-RAYET ANALYSIS}

6.1. APPROXIMATE ANALYSIS - We call an analysis as approximate if only the minimum information is employed which is needed to derive the stellar parameters. The minimal information consists on the equivalent widths of one He I and one He II line and the absolute visual magnitude. The latter value allows to split up the wind density parameter, $p^{W}$, into the radius, $R_{\star}$, and the mass-loss rate, $\dot{M}$. In Fig. 1 we demonstrate this procedure for the star WRT38. Two sets of contour-lines are drawn. These lines correspond to the three observational quantities and mark the loci where theoretical and observed values agree. Each set belongs to a different mass-loss rate: $\dot{M}=10^{-4.4}$ (full drawn) and $10^{-4.0} M_{0} / y r$ (dashed). For neither of the two sets a well defined intersection point results. But by interpolating the contour-lines 


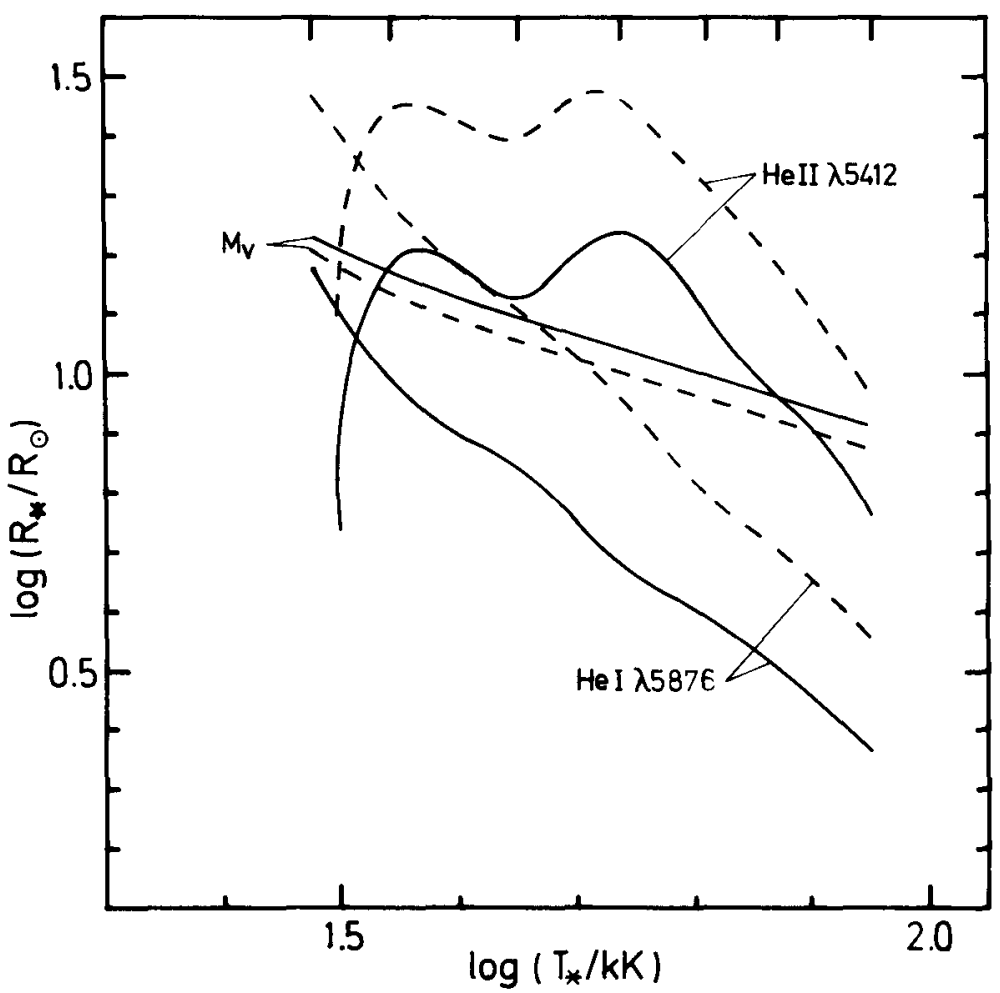

Figure 1. Fit diagram for WRT38, see text (Sect. 6.1)

for a mass-loss rate of about $10^{-4.2} M_{\odot} / y r$, all three lines would join in a single point - the fit point. The model-grid on which the drawn contour-lines are based is calculated with a final wind expansion velocity of $2500 \mathrm{~km} / \mathrm{s}$, while in the case of WR138 the helium line widths correspond to $1500 \mathrm{~km} / \mathrm{s}$. Thus, a model with $T_{\star}=33 \mathrm{kK}$, $R_{\star}=15 R_{\theta}$ and $\dot{M}=10^{-4.5} M_{0} / y r$ reproduces the three observed quantities of WRT38 simultaneously.

6.2. FINE ANALYSIS - The accuracy of an anaiysis may be improved if more than the minimum information is employed. This may be done by fitting the observed line profiles of several helium lines. Though the additional helium lines do not provide more information on the stellar parameters, they can be used as consistency check. More lines help to define the continuum level in crowded spectral regions and to detect line blends. It also improves the confidence to the model calculations if redundant information is reproduced correctly. E.g., after the He I $\lambda \lambda 4471,5876$ and He II $\lambda 5412$ lines of the WN5+abs-A star WR138 have been fitted carefully (Schmutz et al., 1987b), further helium lines are reproduced automatically (Fig. 2). An other example of a fine analysis can be found in Hamann et al. (1987) for the WN5-B star 


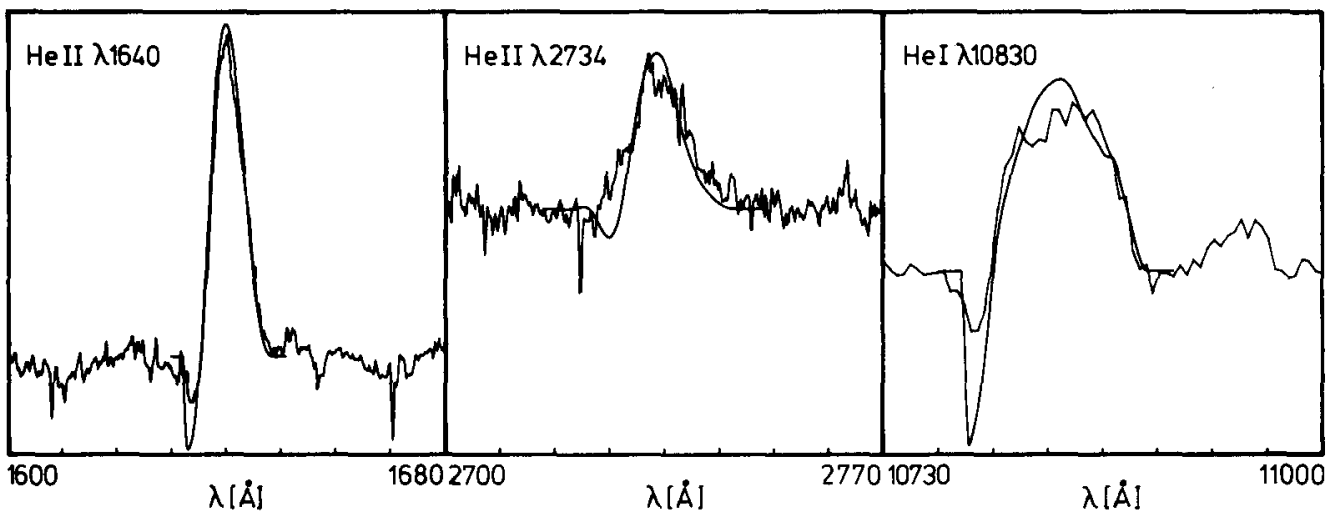

Figure 2. Comparison of calculated helium line profiles to the observed spectrum of the WN5+abs-A star WR138. These lines were not employed in the spectral analyses of this star, but are reproduced automatically by the final model obtained only by fitting the lines He I $\lambda \lambda 4471,5876$ and He II $\lambda 5412$ (Schmutz et al., 1987b)

WR6. In the case of this analysis, the distance to the star is not known and, therefore, its radius, mass-loss rate and luminosity can not be determined. Note that the known radio flux does not provide new information. Both methods, the analysis of the spectral lines and the radio-method, need the knowledge of the distance in order to derive the mass-loss rate. Unluckily, both methods depend on the distance with the same power of the distance, namely $d^{3 / 2}$ ! In the spectral analysis this factor enters by formula 1 in combination with a linear dependence of the stellar radius on the adopted distance; in the radio-method it is contained in the formula of Wright and Barlow (1975).

\section{STELLAR PARAMETERS OF THE WOLF-RAYET STARS}

Approximate analyses as described in Sect. 6.1. have been performed for 30 galactic Wolf-Rayet stars. The resulting stellar parameters $T_{*}, L$ and $\dot{M}$ are given graphically in the Figure 1 of Schmutz et a]. (1987c, these proceedings). In the same contribution the resulting parameters of the Wolf-Rayet stars are discussed briefly. As a check, models with the derived parameters are calculated for some stars and the resulting line profiles are compared with the observations. Two such comparisons are shown in Fig. 3. Dispite of the different classification of the two stars, WN5 and WN3, their helium lines are reproduced by two models which agree in their temperature $T_{*}=90 \mathrm{kK}$. The different spectra emergent from the two models result from the different wind densities. Note that not all WN5 stars are as hot as the example of Fig. 3. An analysis of WR6 (Hamann et al., 1987) yields 60kK and for WRI38 a temperature of only $33 \mathrm{kK}$ is found. This clearly demonstrates the need of a two-dimensional classification. 


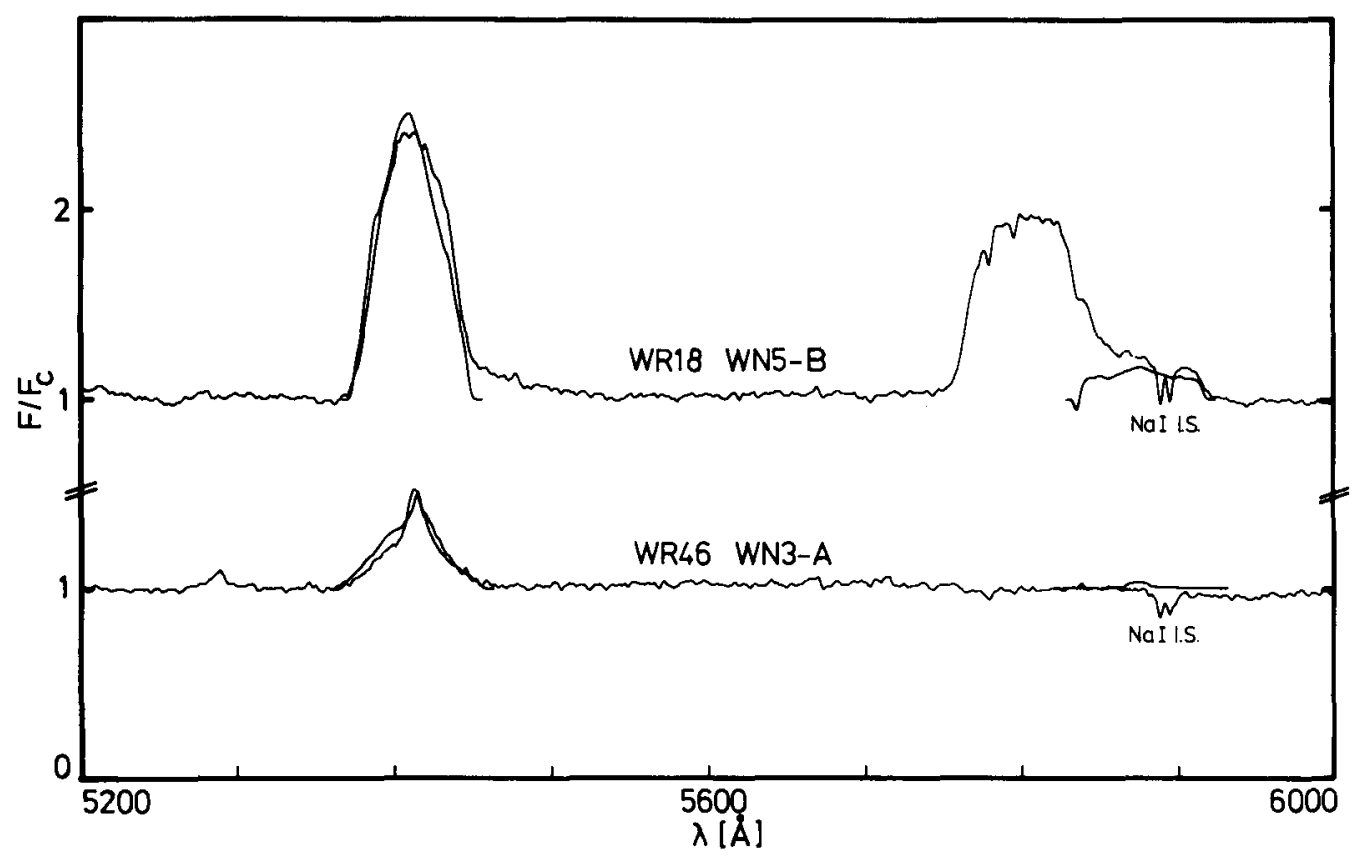

Figure 3. Rectified spectra of WR46, WN3pec-A, and WR18, WN5-B. Superimposed are theoretical line profiles of models with mass-loss rates $\log \left(\dot{M} / \mathrm{M}_{0} / \mathrm{yr}\right)=-4.1$ (top) and -4.9 (botom) and terminal velocities of 2100 and $2500 \mathrm{~km} / \mathrm{s} e \mathrm{c}$, respectively. Otherwise, both models have the same parameters $T_{\star}=90 \mathrm{kK}$ and $R_{\star}=2.5 R_{\theta}$

\section{DISCUSSION}

In Fig. 4 the resulting stellar parameters of the Wolf-Rayet stars are compared with evolutionary tracks (Maeder and Meynet, 1987). It is obvious that the predictions of the evolutionary calculations do not match the stellar parameters of the Wolf-Rayet stars. We are not competent enough to judge, whether it is possible to achieve a better agreement by adjusting the free parameters of the evolution theory. However, we note that the disagreement could be due to the expection that the majority of the Wolf-Rayet stars should belong to the "pealed onion" scenario. There are, in fact, some Wolf-Rayet stars which are $p l a c e d$ in the expected region of the HR-diagram. This holds especially for the hot WNE-A stars which are distinct from the other Wolf-Rayet stars in an important aspect: Their relatively low mass-loss rates of about $10^{-5} M_{\theta} / y r$ ) could be explaind by radiation pressure, provided that the stars are close enough to the Eddington limit (Pauldrach et al., 1985). For all the other Wolf-Rayet stars, particularly for the WC stars, an alternative scenario could apply, concerning their evolutionary status as the mass-loss mechanism. Among the numerous possibilities proposed, the "rotationally induced mixing" (Maeder, 1982), recently explored quantitatively (Maeder, 1987), looks very promisingly. The dashed 


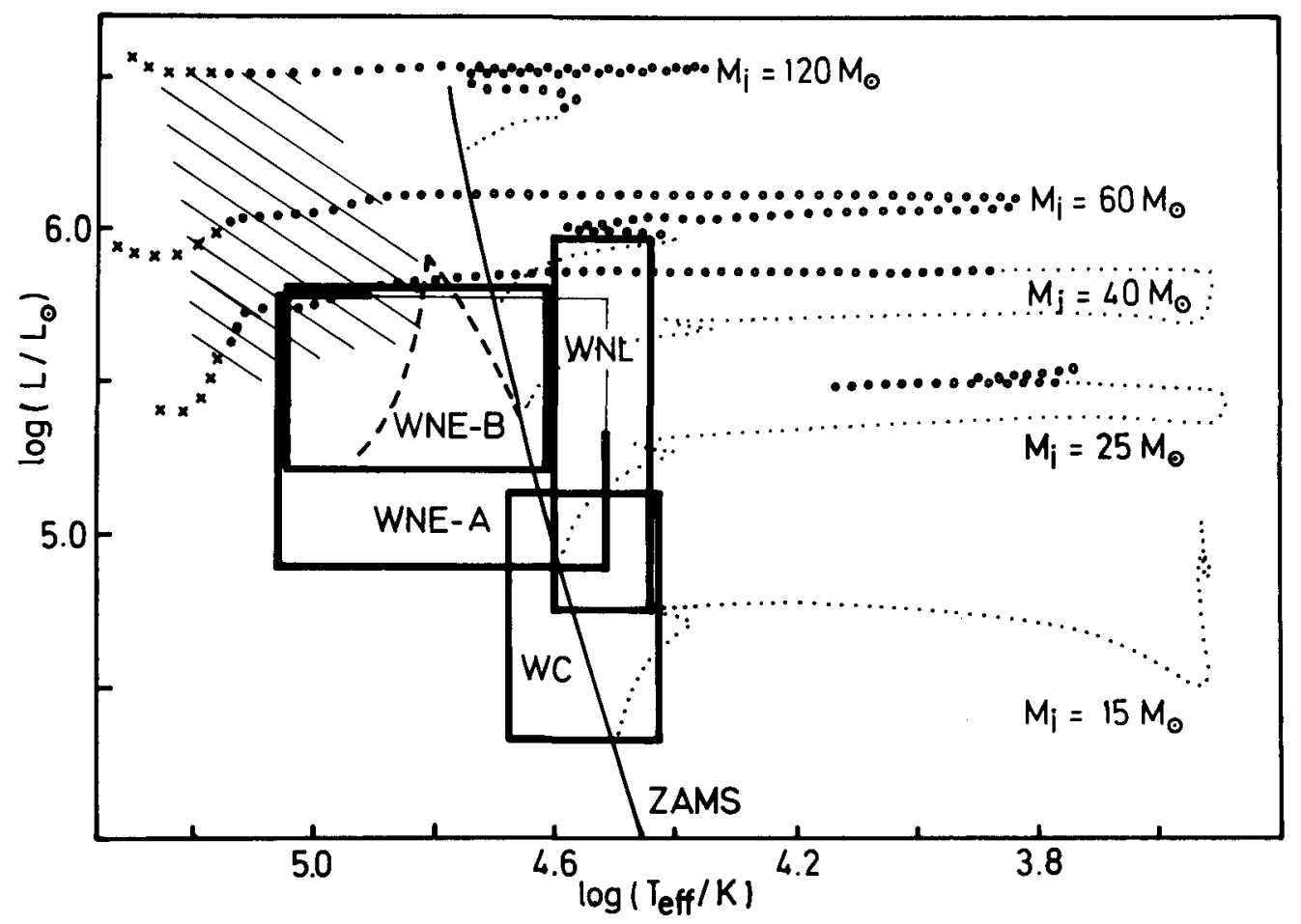

Figure 4. Location of the Wolf-Rayet stars in the HR-diagram superimposed on evolutionary tracks of massive stars computed with mass-loss and overshooting (Maeder and Meynet, 1987). During the phases marked by ' $O$ ' the $\mathrm{C} / \mathrm{N}$ ratio at the surface is about 0.01 , i.e. the star appears to be a WN star; in the phases marked by ' $x$ ' a WC stage would be visible. The hatched area in the upper left part of the diagram indicates where the Wolf-Rayet stars are likely to be observed, considering the evolutionary life-times

track in Fig. 4 is calculated with a rotationally induced turbulence that overcomes the $\mu$-barrier. This track agrees well with the position of the WNE-B stars. The high rotation-velocity of WR138 (Schmutz et al., 1987b) points to rotationally induced mixing, at least for this particular star. A further hint that many Wolf-Rayet stars are not in the post-red-supergiant stage, comes from the inspection of the HRdiagram (Humphreys, 1978) of stellar associations containing Wolf-Rayet stars: Many Wolf-Rayet stars, especially the WNL, are located among luminous association members, which are in the hydrogen burning phase - a fact which can hardly believed to be accidental.

If indeed only a minority of the Wolf-Rayet stars were in the post-red-supergiant phase, the consequences would be twofold: First, the estimate of the ratio of 0-star to Wolf-Rayet life-times, $t_{0} / t_{W R}$, has to be revised, and second, all the indirect nethods used to derive the initial masses of the Wolf-Rayet stars from the age of their surrounding would be not valid (Conti et al., 1983; Schild and Maeder, 1984). 
The second conclusion is based on the fact, that as soon as a star enters the WolfRayet phase, its remaining life-time is extremely short (a few $10^{5}$ years), simply because of the high mass-loss rate. Hence, its total life-time is mainly determined by its age at the onset of the high mass-loss rate. If a star enters the Wolf-Rayet phase very early, or even at zero age, it cannot exist longer than the very massive stars, regardless of its initial mass.

\section{ACKNOWLEDGEMENTS}

All results of the "Kiel-research-group" are obtained in collaboration with Dr. W.-R. Hamann and U. Wessolowski. W.S. greatly appriciates the team-work with his collegues and thanks the DFG and the IAU for travel support. This research was financed by the DFG under grant $\mathrm{Hu}-39 / 25-1$.

\section{REFERENCES}

Castor,J.I., van Blerkom,D.: 1970, Astrophys. J. 161, 485

Conti,P.S., Garmany,C.D., deLoore,C., Vanbeveren,D.: 1983, Astropys. J. 274, 302

Hamann,W.-R.: 1985, Astron. Astrophys. 145, 443

Hamann,W.-R.: 1986, Astron. Astrophys, 160, 347

Hamann,W.-R., Schmutz,W.: 1987, Astron. Astrophys. 174, 173

Hamann,W.-R., Schmutz,W., Wessolowski,U.: 1987, these proceedings and Astron. Astrophys. (in press)

Humphreys, R.M.: 1978, Astrophys. J. Supp1. 38, 309

Hillier,D.J.: 1984, Astrophys. J. 280, 744

Hillier,D.J.: 1986, in 'Luminous Stars and Associations in Galaxies', C.W.H.deLoore, A.J.Willis, P.Laskarides (eds.), IAU Symp. 116, 261

Hillier,D.J.: 1987a, Astrophys. J. Suppl. 63, 947

Hillier,D.J.: 1987b, Astrophys. J. Supp7. 63, 965

Hillier,D.J.: 1987c, Astrophys. J. submitted

Hiltner,W.A., Schild,R.E.: 1966, Astrophys. J. 143, 770

Maeder,A.: 1982, in 'Wolf-Rayet Stars: Observations, Physics, Evolution'

C.W.H.deLoore, A.J.Will is (eds.), IAU Symp. 99, 405

Maeder,A.: 1987, Astron. Astrophys. 178, 159

Maeder,A., Meynet,G.: 1987, Astron. Astrophys. 182, 243

Pauldrach,A., Puls, J, Hummer, D.G., Kudritzki,R.: 1985, Astron. Astrophys. 148, L1

Schild,H., Maeder, A.: 1984, Astron. Astrophys. 136, 237

Schmutz,W.: 1984, in 'Observational Tests of the Stellar Evolution Theory', A.Maeder, A.Renzini (eds.), IAU Symp. 105, 269

Schmutz,W., Hamann,W.-R.: 1986, Astron. Astrophys. Letter 166, L11

Schmutz,W., Hamann,W.-R., Wessolowski,U.: 1987a, in 'Circumstell ar Matter', I.Appenzeller, C.Jordan (eds.), IAU Symp. 122, 461

Schmutz,W., Hamann,W.-R., Wessolowski,U.: 1987b, these proceedings

Schmutz,W., Hamann,W.-R., Wessolowski,U.: 1987b,c, these proceedings and Astron. Astrophys. submitted

Smith,L.J., Willis,A.J.: 1982, Monthly Notices Roy. Astron. Soc. 201, 45$]$

Walborn,N.R.: 1974, Astrophys. J. 189, 269

Wessolowski,U., Schmutz,W., Hamann,W.-R.: 1987, Astron. Astrophys. (in press)

Willis,A.J., Wilson, R.: 1978, Monthly Notices Roy. Astron. Soc. 182, 897

Wright,A.E., Barlow,M.J.: 1975, Monthly Notices Roy. Astron. Soc. 170, 41 